\title{
ON THE FREQUENCY OF SMALL FRACTIONAL PARTS IN CERTAIN REAL SEQUENCES
}

\author{
BY \\ WILLIAM J. LEVEQUE
}

1. Introduction. Let $X_{1}, X_{2}, \cdots$ be a sequence of independent random variables, each uniformly distributed on $[0,1 / 2]$. If $f$ is an arbitrary function from the positive integers to $[0,1 / 2]$, the equation

$$
\operatorname{Pr}\left\{X_{k}<f(k)\right\}=2 f(k)
$$

holds, and it is a consequence of the Borel-Cantelli lemmas [3] that the probability that the inequality $X_{k}<f(k)$ is satisfied for infinitely many $k$ is zero or one, according as the series

$$
\sum_{k=1}^{\infty} f(k)
$$

is convergent or divergent. While it is well known that no such general assertion can be made when the $X_{k}$ are dependent, Khinchin [6] has found a direct analogue in an important case. His theorem is usually stated in measuretheoretic language: the inequality $|k x-p|<f(k)$ has infinitely many integral solutions $k, p$ for almost all $x$ or almost no $x$, according as (2) diverges or converges. We may, however, consider $x$ as a random variable uniformly distributed over some interval, and define the quantity $U_{k}(k=1,2, \cdots)$ as the distance $\langle k x\rangle$ between $k x$ and the nearest integer to $k x$. Then the $U_{k}$ form a sequence of dependent random variables uniformly distributed on $[0,1 / 2]$; Khinchin's theorem shows that the nature of the dependence is not such as to affect the finiteness of the number of solutions of the inequality $U_{k}<f(k)$.

From a probabilistic standpoint the Borel-Cantelli lemmas yield very crude information about a sequence of random variables, and it is of some interest to know whether the $U_{k}$ also resemble the $X_{k}$ in their finer structure. We consider here the case in which (2) diverges, so that there are almost surely infinitely many solutions of $|k x-p|<f(k)$, and investigate in $\S \S 2-3$ the number $T_{n}$ of such solutions with $k \leqq n$. The result is not quite what would be expected from the case of independent variables. For if we put $Y_{k}$ equal to 1 or 0 according as the inequality $X_{k}<f(k)$ does or does not hold, then $S_{n}=Y_{1}+\cdots+Y_{n}$ is the number of $k \leqq n$ such that $X_{k}<f(k)$. Since

Presented to the Society, August 23, 1956; received by the editors September 17, 1956. 


$$
\begin{aligned}
E\left(Y_{k}\right) & =1 \cdot 2 f(k)+0 \cdot(1-2 f(k))=2 f(k), \\
\operatorname{Var} Y_{k} & =E\left(Y_{k}^{2}\right)-E^{2}\left(Y_{k}\right)=2 f(k)-4 f^{2}(k), \\
E\left(S_{n}\right) & =2 \sum_{k=1}^{n} f(k), \\
\operatorname{Var} S_{n} & =2 \sum_{k=1}^{n} f(k)-4 \sum_{k=1}^{n} f^{2}(k),
\end{aligned}
$$

we deduce from the central limit theorem that if $\sum_{1}^{\infty} f^{2}(k)$ converges, then

$$
\lim _{n \rightarrow \infty} \operatorname{Pr}\left\{S_{n}<2 \sum_{k=1}^{n} f(k)+\omega\left(2 \sum_{k=1}^{n} f(k)\right)^{1 / 2}\right\}=\phi(\omega)
$$

where

$$
\phi(\omega)=\frac{1}{(2 \pi)^{1 / 2}} \int_{-\infty}^{\omega} e^{-u^{2} / 2} d u
$$

is the normal distribution function.

The law of the iterated logarithm yields the closely related result that

$$
\operatorname{Pr}\left\{\limsup _{n \rightarrow \infty}\left|\frac{S_{n}-2 \sum_{k=1}^{n} f(k)}{4\left(\sum_{k=1}^{n} f(k) \log \log \sum_{k=1}^{n} f(k)\right)^{1 / 2}}\right|=1\right\}=1
$$

and so in particular

$$
\operatorname{Pr}\left\{S_{n} \sim 2 \sum_{k=1}^{n} f(k)\right\}=1
$$

Theorem 1 exhibits the result corresponding to (3) for $T_{n}$; it differs from (3) in that the coefficient 2 is replaced by $12 \pi^{-2}$.

In $\$ \S 4-6$ we consider the much less strongly dependent sequence $\left\langle r_{1} r_{2} \cdots r_{k} x\right\rangle$, where $r_{1}, r_{2}, \cdots$ is a fixed increasing sequence of positive integers, and show that here the situation is again as described in (3) and (4).

2. A lemma. Let $f$ be a function with the following properties:

$$
\begin{aligned}
& f(x) \text { is positive and decreasing for } x \geqq 0 ; \\
& f(x)=O\left(x^{-1}\right) \text { and } f^{\prime}(x)=O\left(x^{-2}\right) \text { as } x \rightarrow \infty ; \\
& \sum_{k=1}^{\infty} f(k)=\infty .
\end{aligned}
$$


We shall need some further properties of $f$, which we collect in the following lemma.

Lemma 1. If $f$ satisfies (5)-(7) and if $c$ and $\delta$ are positive constants, then

$$
\begin{aligned}
\sum_{k=1}^{n} f(k) & =\int_{1}^{n} f(u) d u+O(1) ; \\
f\left(k+O\left(k^{1-\delta}\right)\right) & =f(k)+O\left(k^{-1-\delta}\right) ; \\
\sum_{k=1}^{c n} f(k) & =\sum_{k=1}^{n} f(k)+O(1) ; \\
\sum_{k=1}^{n} c f(c k) & =\sum_{k=1}^{n} f(k)+O(1) ; \\
\sum_{k=1}^{n} f(k) & =c \sum_{k=1}^{e^{n}} \frac{f(c \log k)}{k}+O(1), \\
\text { if } a_{1}, a_{2}, \cdots \text { and } \alpha \text { are such that } & \sum_{k=1}^{n} a_{k} \sim \text { na }
\end{aligned}
$$

as $n \rightarrow \infty$, then

$$
\sum_{k=1}^{n} a_{k} f(k)=\alpha \sum_{k=1}^{n} f(k)+O(1) .
$$

Part (a) is trivial, and (b) follows from (6) and the law of the mean. Part (c) follows from the estimate

$$
\sum_{k=n}^{c n} f(k)=\sum_{k=n}^{c n} O\left(k^{-1}\right)=O(\log c n-\log n)=O(1),
$$

and (d) from the fact that

$$
\sum_{k=1}^{n} c f(c k)=\int_{1}^{n} c f(c u) d u+O(1)=\int_{c}^{c n} f(t) d t+O(1)=\sum_{k=c}^{c n} f(k)+O(1) .
$$

The substitution $u=c \log v$ in (a) gives (e). To obtain (f), write

$$
\sum_{k=1}^{n}\left(a_{k}-\alpha\right) f(k)=f(n) \sum_{k=1}^{n}\left(a_{k}-\alpha\right)+\sum_{k=1}^{n-1}\left(\sum_{l=1}^{k}\left(a_{l}-\alpha\right)\right)(f(k)-f(k+1))
$$

and note that

$$
f(n) \sum_{k=1}^{n}\left(a_{k}-\alpha\right)=O\left(n^{-1}\right) o(n)=o(1)
$$


and

$$
\begin{aligned}
\sum_{k=1}^{n-1}\left(\sum_{l=1}^{k}\left(a_{l}-\alpha\right)\right)(f(k)-f(k+1)) & =\sum_{k=1}^{n-1} o(k)(f(k)-f(k+1)) \\
& =O(n) \sum_{k=1}^{n-1}(f(k)-f(k+1)) \\
& =O(n f(n))=O(1) .
\end{aligned}
$$

We shall use the following notation: $\mathscr{T}\{A\}$ means the measure of the set of $x \in[0,1]$ such that $A$, if $A$ is a sentence, and it means the measure of $A$ if $A$ is a set.

No $\{m \leqq n \mid \cdots\}$ means the number of positive integers $m \leqq n$ such that....

$E_{x}\{\cdots\}$ or $\{x \mid \cdots\}$ means the set of $x \in[0,1]$ such that $\cdots$.

3. The fractional part of $m x$. We prove the following theorem:

Theоrem 1. Suppose that $f$ satisfies conditions (5)--(7) and put

$$
g(x)=f(\log x) / x .
$$

Let

$$
T_{n}=T_{n}(x)=\operatorname{No}\{m \leqq n \mid\langle m x\rangle<g(m)\} .
$$

Then for fixed $\omega$,

$$
\lim _{n \rightarrow \infty} \mathfrak{T}\left\{T_{n}<\frac{12}{\pi^{2}} \sum_{k=1}^{n} g(k)+\omega\left(\frac{12}{\pi^{2}} \sum_{k=1}^{n} g(k)\right)^{1 / 2}\right\}=\phi(\omega) .
$$

If $x$ is a real number with continued fraction expansion

$$
x=a_{0}+\frac{1}{a_{1}+} \frac{1}{a_{2}+} \cdots=a_{0}+\frac{1}{a_{1}+} \cdots \frac{1}{a_{k}+} \frac{1}{x_{k+1}}
$$

and convergents

$$
\frac{p_{k}}{q_{k}}=a_{0}+\frac{1}{a_{1}+} \cdots \frac{1}{a_{k}},
$$

then

$$
x=\frac{p_{k} x_{k+1}+p_{k-1}}{q_{k} x_{k+1}+q_{k-1}}
$$

and

$$
\left|q_{k} x-p_{k}\right|=\frac{1}{q_{k} \cdot x_{k+1}+q_{k-1}} .
$$


Lemma 2. Put

$$
W_{n}=\operatorname{No}\left\{k \leqq n|| q_{k} x-p_{k} \mid<\frac{f(k)}{q_{k}}\right\} .
$$

Then

$$
\lim _{n \rightarrow \infty} \mathscr{T}\left\{W_{n}<\frac{1}{\log 2} \sum_{k=1}^{n} f(k)+\omega\left(\frac{1}{\log 2} \sum_{k=1}^{n} f(k)\right)^{1 / 2}\right\}=\phi(\omega) .
$$

We take $x$ as a random variable uniformly distributed on $[0,1]$, and use $\operatorname{Pr}_{k}, \mathrm{E}_{k}$ and $\mathrm{Var}_{k}$ to denote conditional probability, expectation and variance when $a_{0}, \cdots, a_{k}$ are given. We suppose throughout this section that $f$ satisfies conditions (5)-(7), and we put $\alpha_{k}=f(k)\left(1+q_{k-1} / q_{k}\right)$ and

$$
V_{k}=\left\{\begin{array}{l}
1-\alpha_{k} \text { if }\left|q_{k} x-p_{k}\right|<\frac{f(k)}{q_{k}}, \\
-\alpha_{k} \text { otherwise. }
\end{array}\right.
$$

Then

$$
\begin{aligned}
\operatorname{Pr}_{k}\left\{V_{k}=1-\alpha_{k}\right\} & =\operatorname{Pr}_{k}\left\{\frac{1}{\left(q_{k} x_{k+1}+q_{k-1}\right)}<\frac{f(k)}{q_{k}}\right\} \\
& =\operatorname{Pr}_{k}\left\{x_{k+1}>\frac{1}{f(k)}-\frac{q_{k-1}}{q_{k}}\right\} \\
& =\operatorname{Pr}_{k}\left\{x \in\left[\frac{p_{k}\left(1 / f(k)-q_{k-1} / q_{k}\right)+p_{k-1}}{q_{k}\left(1 / f(k)-q_{k-1} / q_{k}\right)+q_{k-1}}, \frac{p_{k}}{q_{k}}\right]\right\} \\
& =\frac{\left|\frac{p_{k} q_{k} / f(k) \pm 1}{q_{k}^{2} / f(k)}-\frac{p_{k}}{q_{k}}\right|}{\frac{p_{k}+p_{k-1}}{q_{k}+q_{k-1}}-\frac{p_{k}}{q_{k}} \mid} \\
& =f(k)\left(1+\frac{q_{k-1}}{q_{k}}\right)=\alpha_{k} .
\end{aligned}
$$

Hence

$$
\begin{aligned}
E_{k}\left(V_{k}\right) & =\left(1-\alpha_{k}\right) \alpha_{k}+\left(-\alpha_{k}\right)\left(1-\alpha_{k}\right)=0, \\
\mu_{k}^{2} & =E_{k}\left(V_{k}^{2}\right)=f(k)\left(1+\frac{q_{k-1}}{q_{k}}\right)+O\left(f^{2}(k)\right) .
\end{aligned}
$$

P. Lévy $[9 ; 10$, p. 321$]$ has shown that 


$$
\operatorname{Pr}\left\{\lim _{n \rightarrow \infty} \frac{1}{n} \sum_{k=1}^{n}\left(1+\frac{q_{k-1}}{q_{k}}\right) \sim \frac{1}{\log 2}\right\}=1,
$$

and it follows from (f) of Lemma 1 that for almost all $x$,

$$
\sum_{k=1}^{n} f(k)\left(1+\frac{q_{k-1}}{q_{k}}\right)=\frac{1}{\log 2} \sum_{k=1}^{n} f(k)+O(1) .
$$

Combining (8) and (9), we see that for almost all $x$,

$$
\stackrel{2}{\mu_{1}}+\cdots+\stackrel{2}{2}=\frac{1}{\log 2} \sum_{k=1}^{n} f(k)+O(1) .
$$

We now use a form of the central limit theorem for dependent variables due to Lévy [10, p. 246] (and later extended by J. L. Doob [2, p. 383] as a theorem on martingales):

Lemma 3. Let $Z_{1}, Z_{2}, \cdots$ be a sequence of bounded random variables, and let $E_{n-1}$ denote conditional expectation for given $Z_{1}, \cdots, Z_{n-1}$. Suppose that $E_{n-1}\left(Z_{n}\right)=0$ for $n \geqq 2$, and put

$$
\mu_{n}^{2}=E_{n-1}\left(Z_{n}^{2}\right)=\operatorname{Var}_{n-1}\left(Z_{n}\right) .
$$

For $t>0$, determine $N=N(t)$ so that

$$
\stackrel{2}{\mu_{1}}+\cdots+\stackrel{2}{\mu_{N}} \sim t
$$

and put

$$
S(t)=Z_{1}+\cdots+Z_{N} .
$$

Then if

$$
\operatorname{Pr}\left\{\sum_{n=1}^{\infty} \mu_{n}^{2}<\infty\right\}=0,
$$

we have

$$
\lim _{t \rightarrow \infty} \operatorname{Pr}\left\{\frac{S(t)}{t^{1 / 2}}<\omega\right\}=\phi(\omega) .
$$

If $Z_{k}=V_{k}$, it follows from (10) that aside from a set of measure 0 , the functions $N(t)$ corresponding to various $x$ 's are asymptotically equal, and that

$$
\lim _{n \rightarrow \infty} \operatorname{Pr}\left\{\frac{V_{1}+\cdots+V_{n}}{\left(\frac{1}{\log 2} \sum_{k=1}^{n} f(k)\right)^{1 / 2}}<\omega\right\}=\phi(\omega) .
$$

But 


$$
W_{n}=\sum_{k=1}^{n} V_{k}+\sum_{k=1}^{n} f(k)\left(1+\frac{q_{k-1}}{q_{k}}\right),
$$

and hence for almost all $x$,

$$
W_{n}=\sum_{k=1}^{n} V_{k}+\frac{1}{\log 2} \sum_{k=1}^{n} f(k)+O(1) .
$$

Thus

$$
\lim _{n \rightarrow \infty} \operatorname{Pr}\left\{W_{n}<\frac{1}{\log 2} \sum_{k=1}^{n} f(k)+\omega\left(\frac{1}{\log 2} \sum_{k=1}^{n} f(k)\right)^{1 / 2}\right\}=\phi(\omega),
$$

which completes the proof of the lemma.

The remainder of the proof of Theorem 1 consists in transforming (11) into a statement not involving continued fractions. For this we need an estimate of $q_{k}$.

Lemma 4. If $\delta<1 / 2$, then for almost every $x$ there is a constant $\kappa=\kappa(x, \delta)$ such that

$$
\left|\log q_{k}-\frac{\pi^{2}}{12 \log 2} k\right|<\kappa k^{1-\delta}
$$

This results from an extension of the following theorem of Khinchin [7]: Let $F$ be a function of $k$ positive integral arguments, such that for $n \geqq k$,

$$
\int_{0}^{1} F^{2}\left(a_{n}, \cdots, a_{n-k+1}\right) d x<C,
$$

where $a_{m}=a_{m}(x)$ denotes the mth denominator in the continued fraction expansion of $x$. Then

$$
\lim _{n \rightarrow \infty} \frac{1}{n} \sum_{i=k}^{n} F\left(a_{i}, \cdots, a_{i-k+1}\right)
$$

exists and is constant almost everywhere.

Examination of the proof shows that the theorem may be modified in two ways. The function $F$ may be replaced by a quantity depending on a slowly increasing number of the $a_{m}$; we write

$$
\lim _{n \rightarrow \infty} \frac{1}{n} \sum_{i=1}^{n} F_{i}\left(a_{i}, a_{i-1}, \cdots, a_{i-k_{i}+1}\right),
$$

and require that $i-k_{i}+1$ be positive for $i \geqq 1$. Secondly, the rapidity of approach of the sum in (12) to its limiting value can be estimated by replacing the $\epsilon$ occurring in Khinchin's proof by $n^{-\epsilon}$, where $\epsilon$ is now a sufficiently small 
positive constant. In this way the following theorem can be proved:

Let $\left\{F_{i}\left(r_{1}, \cdots, r_{k_{i}}\right)\right\}$ be non-negative functions of the positive integral arguments $r_{1}, r_{2}, \cdots$, and suppose that the integrals

$$
\int_{0}^{1} F_{i}^{2}\left(a_{i}, a_{i-1}, \cdots, a_{i-k_{i+1}}\right) d x
$$

are uniformly bounded. Suppose further that $\delta<1 / 2$ and that

$$
k_{i}=O\left(\log ^{\sigma} i\right)
$$

for some constant $\sigma>0$. Then there is a constant $B$ such that

$$
\frac{1}{n} \sum_{i=1}^{n} F_{i}\left(a_{i}, \cdots, a_{i-k_{i}+1}\right)=B+O\left(n^{-\delta}\right)
$$

for almost all $x$.

We put

$$
\phi_{i}(x)=a_{i}+\frac{1}{a_{i-1}+} \cdots \frac{1}{a_{i-k_{i}+1}}
$$

and

$$
F_{i}\left(a_{i}, \cdots, a_{i-k_{i+1}}\right)=\log \phi_{i}(x) .
$$

Since $\phi_{i}(x) \leqq a_{i}+1$ and $\mathfrak{M}\left\{a_{i}=r\right\}=\mathfrak{M}\left\{r \leqq x_{i}<r+1\right\}<1 / r^{2}$, we have

$$
\int_{0}^{1} F_{i}^{2} d x \leqq \int_{0}^{1} \log ^{2}\left(a_{i}+1\right) d x \leqq \sum_{r=1}^{\infty} \frac{\log ^{2}(r+1)}{r^{2}} .
$$

Thus for

$$
k_{i}=1+[2 \log i]
$$

there is a $B_{0}$ such that for almost all $x$,

$$
\sum_{i=1}^{n} \log \phi_{i}(x)=B_{0} n+O\left(n^{1-\delta}\right) .
$$

On the other hand, if $\phi_{i}(x)=q_{i} / q_{i-1}$, then by the law of the mean,

$$
\left|\log \phi_{i}(x)-\log \bar{\phi}_{i}(x)\right|=\xi\left|\phi_{i}(x)-\bar{\phi}_{i}(x)\right|
$$

where $\xi<1$. Since

$$
\bar{\phi}_{i}(x)=a_{i}+\frac{1}{a_{i-1}+} \cdots \frac{1}{a_{1}},
$$

this implies that 


$$
\begin{aligned}
& \left|\log \phi_{i}(x)-\log \bar{\phi}_{i}(x)\right| \\
& \quad<\left|\left(a_{i}+\frac{1}{a_{i-1}+} \cdots \frac{1}{a_{i-k_{i}+1}}\right)-\left(a_{i}+\frac{1}{a_{i-1}+} \cdots \frac{1}{a_{i-k_{i}+1}+1}\right)\right|<1 / Q_{k_{i}}^{2},
\end{aligned}
$$

where $P_{l} / Q_{l}$ is the $l$ th convergent in the expansion (13). Since

$$
Q_{l} \geqq Q_{l-1}+Q_{l-2}>2 Q_{l-2}>\cdots>2^{[l / 2]},
$$

we see that

$$
\left|\log \phi_{i}(x)-\log \Phi_{i}(x)\right|<2^{1-k_{i}}<i^{-2 \log 2} .
$$

Thus for almost all $x$,

$$
\sum_{i=1}^{n} \log \phi_{i}(x)=\log q_{n}=B_{0} n+O\left(n^{1-\delta}\right) .
$$

Lévy $\left[10\right.$, p. 320] showed that $B_{0}=\pi^{2} / 12 \log 2$. The proof of Lemma 4 is complete.

Now let

$$
\begin{array}{r}
s_{n}=\text { No }\left\{k \leqq n|| q_{k} x-p_{k} \mid<\frac{f\left(B_{0}^{-1} \log q_{k}\right)}{q_{k}}\right\}, \\
t_{n}(\kappa)=\text { No }\left\{k \leqq n|| q_{k} x-p_{k} \mid<\frac{f\left(k-\kappa k^{1-\delta}\right)}{q_{k}}\right\} .
\end{array}
$$

By (11),

$$
\lim _{n \rightarrow \infty} \mathfrak{T}\left\{t_{n}(\kappa)<\frac{1}{\log 2} \sum_{k=1}^{n} f\left(k-\kappa k^{1-\delta}\right)+\omega\left(\frac{1}{\log 2} \sum_{k=1}^{n} f\left(k-\kappa k^{1-\delta}\right)\right)^{1 / 2}\right\}=\phi(\omega) .
$$

Putting

$$
A_{n}=\frac{1}{\log 2} \sum_{k=1}^{n} f(k)
$$

it follows from (b) of Lemma 1 that for each $\kappa$,

$$
\lim _{n \rightarrow \infty} \mathfrak{T}\left\{t_{n}(\kappa)<A_{n}+\omega A_{n}^{1 / 2}\right\}=\phi(\omega) .
$$

Let

$$
\begin{aligned}
F_{n} & =\left\{x \mid s_{n}<A_{n}+\omega A_{n}^{1 / 2}\right\}, \\
G(\kappa) & =\left\{x|| \log q_{k}-B_{0} k \mid<\kappa k^{1-\delta} \text { for every } k \geqq 1\right\}, \\
H_{n}(\kappa) & =\left\{x \mid t_{n}(\kappa)<A_{n}+\omega A_{n}^{1 / 2}\right\} .
\end{aligned}
$$


Then by Lemma 2 and Equation (14), to each $\epsilon>0$ there corresponds a $\kappa_{0}=\kappa_{0}(\epsilon)$ and an $n_{0}=n_{0}\left(\kappa_{0}, \epsilon\right)=n_{0}(\epsilon)$ such that

$$
\mathfrak{T}\{G(\kappa)\}>1-\epsilon \quad \text { for } \kappa \geqq \kappa_{0}
$$

and

$$
\left|\mathfrak{T}\left\{H_{n}\left( \pm \kappa_{0}\right)\right\}-\phi(\omega)\right|<\epsilon \quad \text { for } n \geqq n_{0} .
$$

Clearly

$$
G\left(\kappa_{0}\right) H_{n}\left(\kappa_{0}\right) \subset F_{n},
$$

and since $\mathfrak{T}(A B) \geqq \mathfrak{T}(A)+\mathfrak{N}(B)-1$ if $A$ and $B$ are subsets of $[0,1]$, we have that for $n \geqq n_{0}$,

$$
\operatorname{Tr}\left\{F_{n}\right\} \geqq 1-\epsilon+\phi(\omega)-\epsilon-1=\phi(\omega)-2 \epsilon .
$$

Similarly, since $G\left(\kappa_{0}\right) F_{n} \subset H_{n}\left(-\kappa_{0}\right)$,

$$
\mathscr{M}\left\{F_{n}\right\} \leqq \phi(\omega)+2 \epsilon .
$$

Hence

$$
\lim _{n \rightarrow \infty} \operatorname{Tr}\left\{F_{n}\right\}=\lim _{n \rightarrow \infty} \operatorname{Pr}\left\{s_{n}<A_{n}+\omega A_{n}^{1 / 2}\right\}=\phi(\omega) .
$$

By the same reasoning we can use (d) of Lemma 1 to show that if

$$
r_{n}=\text { No }\left\{k \leqq n|| q_{k} x-p_{k} \mid<\frac{B_{0} f\left(\log q_{k}\right)}{q_{k}}\right\},
$$

then

$$
\lim _{n \rightarrow \infty} \operatorname{Pr}\left\{r_{n}<A_{n}+\omega A_{n}^{1 / 2}\right\}=\phi(\omega) .
$$

Replacing $f$ by $f / B_{0}$, it follows immediately that

$$
\begin{aligned}
\lim _{n \rightarrow \infty} \operatorname{Pr}\left\{\operatorname{No}\left\{k \leqq n|| q_{k} x-p_{k} \mid<\frac{f\left(\log q_{k}\right)}{q_{k}}\right\}\right. & <\frac{12}{\pi^{2}} \sum_{k=1}^{n} f(k) \\
& \left.+\omega\left(\frac{12}{\pi^{2}} \sum_{k=1}^{n} f(k)\right)^{1 / 2}\right\}=\phi(\omega) .
\end{aligned}
$$

If $|m x-l|<1 / 2 m$, then $l / m$ is a convergent to $x$. Since $f(x)=o(1)$,

No $\left\{k \leqq n|| q_{k} x-p_{k} \mid<\frac{f\left(\log q_{k}\right)}{q_{k}}\right\}$

$$
=\mathrm{No}\left\{m \leqq q_{n} \mid\langle m x\rangle<\frac{f(\log m)}{m}\right\}+O(1),
$$


the error term being uniformly bounded for all $x$. Putting

$$
A(n)=\frac{12}{\pi^{2}} \sum_{k=1}^{e^{n}} \frac{f(\log k)}{k}
$$

and using (e) of Lemma 1 with $c=1$, it follows that

$$
\lim _{n \rightarrow \infty} \operatorname{Pr}\left\{\mathrm{No}\left\{m \leqq q_{n} \mid\langle m x\rangle<\frac{f(\log m)}{m}\right\}<A(n)+\omega A(n)^{1 / 2}\right\}=\phi(\omega) .
$$

There is now a final set-theoretic argument required to eliminate $q_{n}$ entirely. Put

$$
\begin{aligned}
F(n, \omega) & =E_{x}\left\{\mathrm{No}\left\{m \leqq q_{n} \mid\langle m x\rangle<\frac{f(\log m)}{m}\right\}<A(n)+\omega A(n)^{1 / 2}\right\}, \\
G(n, \beta, \omega) & =E_{x}\left\{\mathrm{No}\left\{m \leqq e^{\beta n} \mid\langle m x\rangle<\frac{f(\log m)}{m}\right\}<A(n)+\omega A(n)^{1 / 2}\right\}, \\
H_{N}(\epsilon) & =E_{x}\left\{e^{B_{0}(1-\epsilon) \nu}<q_{\nu}<e^{B_{0}(1+\epsilon) \nu} \text { for all } \nu \geqq N\right\} .
\end{aligned}
$$

It is easily seen that

(16) $H_{N}(\epsilon) G\left(n, B_{0}(1+\epsilon), \omega\right) \subset F(n, \omega), H_{N}(\epsilon) F(n, \omega) \subset H_{N}(\epsilon) G\left(n, B_{0}(1-\epsilon), \omega\right)$ for $0<\epsilon<1, n \geqq N$. On the other hand, we have

$$
\begin{aligned}
G\left(\frac{1-\epsilon}{1+\epsilon} n, B_{0}(1+\epsilon), \eta\right)=E_{x}\{\text { No }\{m & \left.\leqq e^{B_{0}(1-\epsilon) n} \mid\langle m x\rangle<\frac{f(\log m)}{m}\right\} \\
& \left.<A\left(\frac{1-\epsilon}{1+\epsilon} n\right)+\eta A^{1 / 2}\left(\frac{1-\epsilon}{1+\epsilon} n\right)\right\},
\end{aligned}
$$

and hence if $\eta$ is chosen so that

$$
A\left(\frac{1-\epsilon}{1+\epsilon} n\right)+\eta A^{1 / 2}\left(\frac{1-\epsilon}{1+\epsilon} n\right)>A(n)+\omega A^{1 / 2}(n),
$$

then

$$
G\left(\frac{1-\epsilon}{1+\epsilon} n, B_{0}(1+\epsilon), \eta\right) \supset G\left(n, B_{0}(1-\epsilon), \omega\right) .
$$

By (c) of Lemma $1, A(c n)=A(n)+O(1)$, so

$$
A\left(\frac{1-\epsilon}{1+\epsilon} n\right)+\eta A^{1 / 2}\left(\frac{1-\epsilon}{1+\epsilon} n\right)=A(n)+\left(\eta+O\left(A^{-1 / 2}(n)\right)\right) A^{1 / 2}(n) .
$$

Since $A(n) \rightarrow \infty$ as $n \rightarrow \infty$, it follows that if $\delta>0$ is arbitrary, (17) holds with $\eta=\omega+\delta$, if $n>n_{0}(\epsilon, \delta)$. But then by (16) and (18), 


$$
H_{N}(\epsilon) F(n, \omega) \subset H_{N}(\epsilon) G\left(n, B_{0}(1-\epsilon), \omega\right) \subset F\left(\frac{1-\epsilon}{1+\epsilon} n, \omega+\delta\right)
$$

for

$$
n>\min \left(\frac{1+\epsilon}{1-\epsilon} N, n_{0}\right) .
$$

By Lemma $4, \mathfrak{T}\left\{H_{N}(\epsilon)\right\} \rightarrow 1$ as $N \rightarrow \infty$, and by (15), $\mathfrak{N}\{F(n, \omega)\} \rightarrow \phi(\omega)$ as $n \rightarrow \infty$. Hence, if we allow $n$ and $N$ to increase in such a way that

$$
N(1+\epsilon) /(1-\epsilon)<n,
$$

we obtain the inequality

$$
\phi(\omega) \leqq \lim _{n \rightarrow \infty} \mathfrak{T}\left\{G\left(n, B_{0}(1-\epsilon), \omega\right)\right\} \leqq \phi(\omega+\delta) .
$$

Since $\delta$ is arbitrary and $\phi$ is continuous,

$$
\lim _{n \rightarrow \infty} \operatorname{Tr}\left\{G\left(n, B_{0}(1-\epsilon), \omega\right)\right\}=\phi(\omega) .
$$

Since $\epsilon$ is arbitrary (in $[0,1]$ ), we can choose $\epsilon=1-B_{0}^{-1}$, and obtain

$$
\lim _{n \rightarrow \infty} \mathfrak{T}\{G(n, 1, \omega)\}=\phi(\omega),
$$

or

$$
\begin{aligned}
\lim _{n \rightarrow \infty} \operatorname{Pr}\left\{N _ { 0 } \left\{m \leqq e^{n} \mid\langle m x\rangle\right.\right. & \left.<\frac{f(\log m)}{m}\right\} \\
& \left.<\frac{12}{\pi^{2}} \sum_{k=1}^{e^{n}} \frac{f(\log k)}{k}+\omega\left(\frac{12}{\pi^{2}} \sum_{k=1}^{e n} \frac{f(\log k)}{k}\right)^{1 / 2}\right\}=\phi(\omega) .
\end{aligned}
$$

Using (c) of Lemma 1 again (with $1 \leqq c \leqq(n+1) / n$ ) and the fact that there are at most three denominators $q_{k}$ lying between $e^{n}$ and $e^{n+1}$, we obtain Theorem 1.

4. The small values of $\left\langle r_{1} r_{2} \cdots r_{n} x\right\rangle$. We now consider sequences of the form $\left\langle r_{1} r_{2} \cdots r_{n} x\right\rangle$, where $x$ is again uniformly distributed on $[0,1]$ and $r_{1}, r_{2}, \cdots$ is a fixed nondecreasing sequence of integers larger than 1 , not depending on $x$, with $\lim r_{n}=\infty$. Let the sequences $\left\{x_{n}\right\}$ and $\left\{a_{n}\right\}$ of real numbers and integers, respectively, be determined by the following conditions:

$$
\begin{gathered}
r_{1} x=a_{1}+x_{1}, \quad-1 / 2 \leqq x_{1}<1 / 2, \\
r_{2} x_{1}=a_{2}+x_{2}, \quad-1 / 2 \leqq x_{2}<1 / 2, \\
\cdot \cdot \cdot \cdot \cdot \cdot \cdot \cdot \cdot \cdot \cdot \cdot \\
r_{n} x_{n-1}=a_{n}+x_{n}, \quad-1 / 2 \leqq x_{n}<1 / 2,
\end{gathered}
$$


Then

$$
\begin{gathered}
a_{n}=\left[r_{n} x_{n-1}+1 / 2\right], \quad\left|x_{n}\right|=\left\langle r_{n} x_{n-1}\right\rangle, \\
-\left[\frac{r_{n}}{2}\right] \leqq a_{n}<\left[\frac{r_{n}}{2}\right]
\end{gathered}
$$

for $n=1,2, \cdots$, and

$$
x=\sum_{n=1}^{\infty} \frac{a_{n}}{r_{1} \cdots r_{n}} .
$$

The series (21) bears an obvious relation to the expansion of $x$ to the base $r$ if, contrary to assumption, we take all $r_{n}=r$, and to the Cantor factorial expansion if $r_{n}=n$ for all $n$. In any case, the expansion is unique except for a set of measure zero.

Since $x$ is a random variable, so is every element of $\left\{x_{n}\right\}$ and $\left\{a_{n}\right\}$, and it is easily seen that each $x_{n}$ is uniformly distributed on $[-1 / 2,1 / 2]$, and that each $a_{n}$ is discretely uniformly distributed, in the sense that

$$
\operatorname{Pr}\left\{a_{n}=j\right\}=\frac{1}{r_{n}} \text { for } \quad-\left[\frac{r_{n}}{2}\right] \leqq j<\left[\frac{r_{n}}{2}\right] .
$$

There is a significant difference between the two sets of variables, however, in that the $a_{n}$ are statistically independent, while the $x_{n}$ are not, as the Equations (19) show. Dependence makes the sequence $\left\{x_{n}\right\}$ difficult to analyze probabilistically, but a considerable amount of information can be gained indirectly by transferring results about $\left\{a_{n}\right\}$ via the relation

$$
x_{n-1}=\frac{a_{n}}{r_{n}}+O\left(\frac{1}{r_{n}}\right) .
$$

TheOREM 2. Suppose that $r_{1}, r_{2}, \ldots$ is a nondecreasing sequence of positive integers such that $r_{n}^{m}>n$ for some fixed integer $m$. Let $R_{n}=r_{1} r_{2} \cdots r_{n}$, and let $f$ be a positive function. Let $S$ be an increasing sequence of positive integers. Then the inequality

$$
\left\langle R_{n} x\right\rangle<f(n)
$$

has infinitely many solutions $n \in S$ for almost all $x$ or almost no $x$, according as the series

$$
\sum_{n \in S} f(n)
$$

diverges or converges.

We note first that it suffices to consider functions $f$ such that $f(n) \geqq n^{-2}$ for all $n \in S$. For if (24) converges, then so does the series 


$$
\sum_{n \in S} f^{*}(n)
$$

where

$$
f^{*}(n)=\left\{\begin{array}{l}
f(n) \text { if } f(n) \geqq n^{-2}, \\
n^{-2} \text { otherwise, }
\end{array}\right.
$$

and if the inequality $\left\langle R_{n} x\right\rangle\left\langle f^{*}(n)\right.$ has only finitely many solutions in $S$, the same is surely true of (23). Suppose on the other hand that (24) diverges. Then so also does

$$
\sum f\left(n_{j}\right)
$$

the summation being extended over the integers $n_{j} \in S$ such that $f\left(n_{j}\right) \geqq n_{j}^{-2}$. These integers constitute a subsequence $S^{\prime}$ of $S$, and the truth of the theorem for $S^{\prime}$ implies its truth for $S$.

We suppose throughout the proof that $n \in S$. If we put

$$
P_{n}=R_{n} \sum_{j=1}^{n} \frac{a_{j}}{R_{j}}
$$

then

$$
\left|R_{n} x-P_{n}\right|=\left|x_{n}\right| \leqq 1 / 2
$$

so

$$
\left|R_{n} x-P_{n}\right|=\left\langle R_{n} x\right\rangle .
$$

For each $n$ let $k_{n}$ be the unique positive integer such that

$$
\left[r_{n+1} \cdots r_{n+k_{n}-1} f(n)+1 / 2\right]=0, \quad\left[r_{n+1} \cdots r_{n+k_{n}} f(n)+1 / 2\right] \neq 0 ;
$$

in particular, if $\left[r_{n+1} f(n)+1 / 2\right] \neq 0$ then $k_{n}=1$. Then

$$
\frac{1}{r_{n+1} \cdots r_{n+k_{n}}} \leqq 2 f(n) \text {. }
$$

Let $\varepsilon_{n}$ be the event that (i.e., the set of $x \in[0,1]$ such that) $a_{n+1}=\cdots=a_{n+k_{n}-1}=0,\left|a_{n+k_{n}}\right|<r_{n+1} \cdots r_{n+k_{n}} f(n)+1=\frac{R_{n+k_{n}}}{R_{n}} f(n)+1$, and for $c>0$ let $\mathcal{F}_{n}(c)$ be the event that $\left\langle R_{n} x\right\rangle\langle c f(n)$.

Suppose that $x \in \mathcal{F}_{n}(1)$. If $k_{n}=1$, then we have

$$
\begin{gathered}
\left|x_{n}\right|<f(n), \\
\left|a_{n+1}\right|=\left|a_{n+k_{n}}\right|=\left|\left[r_{n+1} x_{n}+1 / 2\right]\right| \leqq r_{n+1}\left|x_{n}\right|+1 / 2<r_{n+k_{n}} f(n)+1,
\end{gathered}
$$

so $x \in \varepsilon_{n}$. If $k_{n}>1$, then 


$$
\begin{aligned}
&\left|a_{n+1}\right| \leqq\left[r_{n+1} f(n)+1 / 2\right]=0, \quad x_{n+1}=r_{n+1} x \\
&\left|a_{n+2}\right| \leqq\left[r_{n+1} r_{n+2} f(n)+1 / 2\right]=0, \quad x_{n+2}=r_{n+1} r_{n+2} x_{n}, \\
& \cdot \cdot \cdot \cdot \cdot \cdot \cdot \cdot \cdot \cdot \cdot \cdot \cdot \cdot \cdot \cdot \cdot \\
&\left|a_{n+k_{n}-1}\right| \leqq\left[r_{n+1} \cdots r_{n+k_{n}-1} f(n)+1 / 2\right]=0, x_{n+k_{n}-1}=r_{n+1} \cdots r_{n+k_{n-1} x_{n}}, \\
&\left|a_{n+k_{n}}\right| \leqq\left[r_{n+1} \cdots r_{n+k_{n}} f(n)+1 / 2\right]<r_{n+1} \cdots r_{n+k_{n}} f(n)+1,
\end{aligned}
$$

and again $x \in \mathcal{E}_{n}$. Hence $\mathcal{F}_{n}(1) \subset \mathcal{E}_{n}$.

On the other hand, if $x \in \mathcal{E}_{n}$ then

$$
x=\sum_{j=1}^{n} \frac{a_{j}}{R_{j}}+\sum_{j=n+k_{n}}^{\infty} \frac{a_{j}}{R_{j}}
$$

so

$$
\begin{aligned}
\left\langle R_{n} x\right\rangle & =\left|R_{n} x-P_{n}\right|<\frac{\left(\left|a_{n+k_{n}}\right|+1\right) R_{n}}{R_{n+k_{n}}}<\frac{\left(\frac{R_{n+k_{n}}}{R_{n}} f(n)+2\right) R_{n}}{R_{n+k_{n}}} \\
& =f(n)+\frac{2 R_{n}}{R_{n+k_{n}}}
\end{aligned}
$$

and it follows from (26) that $\varepsilon_{n} \subset \mathcal{F}_{n}(3)$.

Thus if $\varepsilon_{n}$ occurs for only finitely many $n \in S$, the same is true of $\mathcal{F}_{n}(1)$; while if $\mathcal{E}_{n}$ occurs for infinitely many $n \in S$, the same is true of $\mathcal{F}_{n}(3)$. Since the convergence of $(24)$ is unaffected by replacing $f(n)$ by $3 f(n)$, there remains only the task of showing that $\mathcal{E}_{n}$ occurs for infinitely many $n \in S$, or only finitely many $n \in S$, for almost all $x$, according as (24) diverges or converges.

Since $r_{n}^{m}>n$ and $f(n)>n^{-2}$, we have $r_{n+1} \cdots r_{n+2 m} f(n)>1$. Hence $k_{n} \leqq 2 m$, and the event $\varepsilon_{n}$ depends on at most the $2 m$ random variables $a_{n+1}, \cdots$, $a_{n+2 m}$. Hence for fixed $l(0 \leqq l<2 m)$, the events $\varepsilon_{2 v m+l}(\nu=0,1, \cdots)$ are independent. By (22),

$$
\operatorname{Pr}\left\{\left|a_{n}\right|=j\right\}= \begin{cases}\frac{1}{r_{n}} & \text { if } j=0, \\ \frac{2}{r_{n}} & \text { if } 0<j<\frac{r_{n}}{2}, \\ \frac{1}{r_{n}} & \text { if } j=\frac{r_{n}}{2}, \quad r_{n} \text { even. }\end{cases}
$$

Hence for arbitrary real $u \in\left[0, r_{n} / 2\right)$,

$$
\operatorname{Pr}\left\{\left|a_{n}\right| \leqq u\right\}=\frac{2[u]+1}{r_{n}}\left\{\begin{array}{l}
\leqq(2 u+1) / r_{n}, \\
\geqq(2 u-1) / r_{n} .
\end{array}\right.
$$


Thus, because of the independence of the $a_{n}$, we have

$$
\operatorname{Pr}\left\{\varepsilon_{n}\right\} \leqq \frac{1}{r_{n+1}} \cdots \frac{1}{r_{n+k_{n}-1}} \cdot \frac{2 \frac{R_{n+k_{n}}}{R_{n}} f(n)+3}{r_{n+k_{n}}}=2 f(n)+\frac{3 R_{n}}{R_{n+k_{n}}},
$$

and by (26),

$$
\operatorname{Pr}\left\{\varepsilon_{n}\right\}<8 f(n)
$$

Also

$$
\operatorname{Pr}\left\{\varepsilon_{n}\right\} \geqq \frac{2 \frac{R_{n+k_{n}}}{R_{n}} f(n)+1}{r_{n+1} \cdots r_{n+k_{n}}}>2 f(n) .
$$

Hence for each $l$ the series $\left({ }^{1}\right)$

$$
\sum_{\nu ; 2 v m+l \in S} \operatorname{Pr}\left\{\varepsilon_{2 v m+l}\right\}
$$

converges or diverges with the series

$$
\sum_{\nu ; 2 v m+l \in S} f(2 v m+l) .
$$

But if the series

$$
\sum_{n \in S} f(n)
$$

diverges, at least one of the series (30), for $0 \leqq l<2 m$, must diverge, while if (31) converges, all the series (30) converge. The theorem therefore follows from the Borel-Cantelli lemmas.

5. We now consider the case in which (23) has infinitely many solutions for almost all $x$, and investigate the number of such solutions with $n \leqq N$. For simplicity we suppose that $S$ is the full set of positive integers.

TheOREM 3. Let $\left\{r_{n}\right\}$ and $\left\{R_{n}\right\}$ be as described in Theorem 2. Let $f$ be a positive function such that

$$
\sum_{n=1}^{\infty} f(n)=\infty, \quad f(n)=O\left(n^{-1 / 2-\epsilon}\right) .
$$

Let $k_{n}$ be the positive integer defined in (25), and suppose that

$$
\sum_{n=1}^{\infty}\left(r_{n+1} \cdots r_{n+k_{n}}\right)^{-1}<\infty .
$$

(1) The symbol $\sum_{v_{i}} \ldots$ means summation over those $\nu$ such that $\cdots$. 
Then

$$
\begin{aligned}
\lim _{N \rightarrow \infty} \operatorname{Pr}\left\{\mathrm { No } \left\{n \leqq N \mid\left\langle R_{n} x\right\rangle\right.\right. & <f(n)\} \\
& \left.<2 \sum_{n=1}^{N} f(n)+\omega\left(2 \sum_{n=1}^{N} f(n)\right)^{1 / 2}\right\}=\phi(\omega) .
\end{aligned}
$$

According to Theorem 2, the $n$ for which $f(n)<n^{-2}$ contribute only a bounded number of solutions of the inequality (23), so we may suppose that $f(n) \geqq n^{-2}$. Put

$$
X_{n}= \begin{cases}1 \text { if }\left\langle R_{n} x\right\rangle<f(n) \\ 0 \text { otherwise }\end{cases}
$$

and

$$
S_{N}=\sum_{n=1}^{N} X_{n}
$$

Similarly, put

$$
Y_{n}=\left\{\begin{array}{l}
1 \text { if } \varepsilon_{n} \text { occurs } \\
0 \text { otherwise }
\end{array}\right.
$$

and

$$
T_{N}=\sum_{n=1}^{N} Y_{n}
$$

where $\mathcal{E}_{n}$ has the same meaning as before. Since $F_{n}(1) \subset \mathcal{E}_{n}$, we have

$$
S_{N}<T_{N} .
$$

On the other hand, if $Y_{n}=1$ then either $X_{n}=1$ or

$$
\left\langle R_{n} x\right\rangle \in\left[f(n), f(n)+\frac{2 R_{n}}{R_{n+k_{n}}}\right],
$$

by (27). Because of the uniform distribution of the $x_{n}$, the probability of the event (35) is $2 R_{n} / R_{n+k_{n}}$, and by (32) and the first Borel-Cantelli lemma, the event (35) occurs only finitely many times, for almost all $x$. Thus given $\epsilon>0$, there is a constant $M$ so large that

$$
T_{N}<S_{N}+M
$$

for all $N$ and all $x$ not in a set of measure at most $\epsilon$. Combining (34) and (36), we see that (33) will follow if it can be shown that 


$$
\lim _{N \rightarrow \infty} \operatorname{Pr}\left\{T_{N}<2 \sum_{n=1}^{N} f(n)+\omega\left(2 \sum_{n=1}^{N} f(n)\right)^{1 / 2}\right\}=\phi(\omega) .
$$

To this end we first prove a general lemma, suggested by work of Hoeffding and Robbins [5]. A set of random variables $Z_{1}, Z_{2}, \cdots$ is said to be $m$-dependent if for every $r, s$ and $n$ for which $n>s>r+m$, the sets $Z_{1}, \cdots, Z_{r}$ and $Z_{s}, \cdots, Z_{n}$ are independent. (The variables $Y_{n}$ above are $2 m$-dependent.)

Theorem 4. Let $Z_{1}, Z_{2}, \cdots$ be a sequence of $m$-dependent random variables such that

$$
Z_{n}=\left\{\begin{array}{l}
1 \text { with probability } p_{n} \\
0 \text { with probability } 1-p_{n}
\end{array}\right.
$$

Suppose that

$$
\begin{gathered}
\sum_{n=1}^{\infty} p_{n}=\infty, \\
p_{n}=O\left(n^{-1 / 2-\epsilon}\right), \quad \epsilon>0, \\
\sum_{i=1}^{\infty} \sum_{j=1}^{\infty}\left|\operatorname{Cov}\left(Z_{i}, Z_{i+j}\right)\right|<\infty .
\end{gathered}
$$

Then

$$
\lim _{n \rightarrow \infty} \operatorname{Pr}\left\{Z_{1}+\cdots+Z_{n}<\sum_{k=1}^{n} p_{k}+\omega\left(\sum_{k=1}^{n} p_{k}\right)^{1 / 2}\right\}=\phi(\omega) .
$$

We decompose the finite sequence $1,2, \cdots, n$ into blocks, in the following way. Choose $\eta$ smaller than $\epsilon$, and find an integer $l_{0}$ such that

$$
\left(l_{0}+1\right)^{2+\eta}-l_{0}^{2+\eta}>2 m \text {. }
$$

For $q \geqq 1$ put

$$
l_{q}=\left[\left(l_{0}+q\right)^{2+\eta}\right],
$$

and define $\kappa=\kappa(n)$ by the inequality

$$
l_{\kappa} \leqq n<l_{\kappa+1} \text {. }
$$

For $1 \leqq q<\kappa-1$, let $I_{q+1}$ be the set of integers $j$ such that $l_{q}<j \leqq l_{q+1}-m$, and let $J_{q+1}$ be the set of integers $j$ such that $l_{q+1}-m<j \leqq l_{q+1}$. Finally, put

$$
\begin{aligned}
U_{q} & =\sum_{\nu \in I_{q}} Z_{\nu}=\sum_{I_{q}} Z_{\nu}, \\
V_{q} & =\sum_{J_{q}} Z_{\nu},
\end{aligned}
$$


for $q=2, \cdots, \kappa$, so that

$$
Q_{n}=\sum_{\nu=1}^{n} Z_{\nu}=\sum_{\nu=1}^{l_{1}} Z_{\nu}+\sum_{q=2}^{\kappa} U_{q}+\sum_{q=2}^{\kappa} V_{q}+\sum_{\nu=l_{k}+1}^{n} Z_{\nu} .
$$

By the definitions of $l_{0}$ and $m$-dependence, the variables $U_{2}, \cdots, U_{k}$ are independent, as are $V_{2}, \cdots, V_{k}$. We shall show that the limiting behavior of $Q_{n}$ is determined by that of $\sum U_{q}$, and then apply a standard version of the central limit theorem.

Since $l_{1}$ is fixed and the $Z$ 's are bounded, the sum

$$
\sum_{\nu=1}^{l_{1}} Z_{\nu}
$$

is clearly negligible in the limit, if $\operatorname{Var}\left(S_{n}\right) \rightarrow \infty$. By (40), (39), and (38),

$$
\begin{aligned}
\operatorname{Var}\left(\sum_{q=2}^{\kappa} V_{q}\right) & =\sum_{q=2}^{\kappa} \sum_{J_{q}} \operatorname{Var}\left(Z_{\nu}\right)+2 \sum_{q=2}^{\kappa} \sum_{J_{q}} \operatorname{Cov}\left(Z_{\mu}, Z_{\nu}\right) \\
& =\sum_{q=2}^{\kappa} \sum_{J_{q}}\left(p_{\nu}-p_{\nu}^{2}\right)+O(1) \\
& =\sum_{q=2}^{\kappa} \sum_{J_{q}} p_{\nu}+O(1) \\
& =\sum_{q=2}^{\kappa} \sum_{\nu=1}^{n} O\left(l_{q}^{-1 / 2-\epsilon}\right)+O(1) \\
& =O\left(\sum_{q=2}^{\kappa} q^{-1-2 \epsilon-\eta / 2-\epsilon \eta}\right)+O(1)
\end{aligned}
$$

so that

$$
\operatorname{Var}\left(\sum_{q=2}^{k} V_{q}\right)=O(1)
$$

Turning to $U_{q}$, we see that

and

$$
E\left(U_{q}\right)=\sum_{I_{q}} p_{v}=e_{q}
$$

$$
\operatorname{Var}\left(U_{q}\right)=\sum_{I} \operatorname{Var}\left(Z_{\nu}\right)+2 \sum_{\mu, \nu \in I_{q} ; \mu<\nu<\mu+m} \operatorname{Cov}\left(Z_{\mu}, Z_{\nu}\right),
$$

so that

$$
\sigma_{\kappa}^{2}=\operatorname{Var}\left(U_{2}+\cdots+U_{\kappa}\right)=\sum_{q=2}^{\kappa} e_{q}+O(1) .
$$


Now

$$
\begin{aligned}
e_{q} & <c \sum_{I_{q}} \frac{1}{\nu^{1 / 2+\epsilon}}<c\left(l_{q}^{1 / 2-\epsilon}-l_{q-1}^{1 / 2-\epsilon}\right) \\
& <c l_{q-1}^{1 / 2-\epsilon}\left\{\left(1+\frac{1}{q}\right)^{(1 / 2-\epsilon)(2+\eta)}-1\right\} \\
& =O\left(q^{(2+\eta)(1 / 2-\epsilon)} \cdot \frac{1}{q}\right)
\end{aligned}
$$

and so

$$
e_{q}=O(1)
$$

This implies in particular that

$$
\operatorname{Var}\left(\sum_{\nu=l_{\kappa}+1}^{n} Z_{\nu}\right)=O(1)
$$

and hence, since

$$
\sum_{q=2}^{\infty} \sum_{J_{q}} p_{\nu}<\infty
$$

that

$$
\sigma_{\kappa}^{2}=\sum_{\nu=1}^{n} p_{\nu}+O(1), \quad E\left(U_{2}+\cdots+U_{\kappa}\right)=\sum_{\nu=1}^{n} p_{\nu}+O(1) .
$$

If we put

$$
\pi_{n}=\sum_{\nu=1}^{n} p_{\nu}
$$

then (42) shows that

$$
\operatorname{Var}\left(\pi_{n}^{-1 / 2} \sum_{q=2}^{\kappa} V_{q}\right)=O(1)
$$

and it follows from Chebyshev's inequality that the random variable $\pi_{n}^{-1 / 2} \sum_{2}^{\kappa} V_{q}$ approaches zero in probability. By the same reasoning this is true also of $\pi_{n}^{-1 / 2} \sum_{1}^{l_{1}} Z_{\nu}$. Combining these facts with (46), we see $[1$, p. 254] that the limiting distribution of $\left(Q_{n}-\pi_{n}\right) / \pi_{n}^{1 / 2}$ is identical with that of

$$
\left(U_{2}+\cdots+U_{\kappa}-\pi_{n}\right) / \pi_{n}^{1 / 2} .
$$

We now wish to apply Iyapunov's criterion $[1$, p. 213], according to which the normalized sum (48) is asymptotically normal, with mean zero and variance 1 , if 


$$
\left(\sum_{q=2}^{\kappa} \rho_{q}^{3}\right)^{1 / 3}=O\left(\sigma_{\kappa}\right)
$$

where

$$
\rho_{q}^{3}=E\left(\left|U_{q}-E\left(U_{q}\right)\right|^{3}\right) .
$$

This will complete the proof of Theorem 4 . We have

$$
\begin{aligned}
\rho_{q}^{3} & \leqq E\left\{\left(\sum_{I_{q}}\left|Z_{\nu}-p_{\nu}\right|\right)^{3}\right\} \\
<6 E\left\{\sum_{\nu \in I_{q}}\left|Z_{\nu}-p_{\nu}\right|^{3}\right. & +\sum_{\mu, \nu \in I_{q}}\left|Z_{\mu}-p_{\mu}\right| \cdot\left|Z_{\nu}-p_{\nu}\right|^{2} \\
& \left.+\sum_{\mu, \nu, \lambda \in I_{q}}\left|Z_{\mu}-p_{\mu}\right| \cdot\left|Z_{\nu}-p_{\nu}\right| \cdot\left|Z_{\lambda}-p_{\lambda}\right|\right\} .
\end{aligned}
$$

Now

$$
\begin{aligned}
\sum_{I_{q}} E\left(\left|Z_{\nu}-p_{\nu}\right|^{3}\right) & =\sum_{I_{q}}\left(1-p_{\nu}\right)^{3} p_{\nu}+\sum_{I_{q}} p_{\nu}^{3}\left(1-p_{\nu}\right) \\
& =e_{q}+O\left(\sum_{I_{q}} p_{\nu}^{2}\right) .
\end{aligned}
$$

Since $\left|Z_{\nu}-p_{\nu}\right|<1$, we have, by the generalized Hölder inequality [4, p. 140],

$$
\begin{gathered}
\sum_{\mu, \nu \in I_{q}} E\left(\left|Z_{\mu}-p_{\mu}\right| \cdot\left|Z_{\nu}-p_{\nu}\right|^{2}\right) \leqq \sum_{\mu, \nu \in I_{q}} E\left(\left|Z_{\mu}-p_{\mu}\right| \cdot\left|Z_{\nu}-p_{\nu}\right|\right) \\
\leqq\left(\sum_{\mu, \nu \in I_{q}} \operatorname{Var}\left(Z_{\mu}\right) \operatorname{Var}\left(Z_{\nu}\right)\right)^{1 / 2} \leqq \sum_{\mu \in I_{q}} \operatorname{Var}\left(Z_{\mu}\right) \\
=\sum_{\mu \in I_{q}}\left(p_{\mu}-p_{\mu}^{2}\right)=e_{q}+O\left(\sum_{I_{q}} p_{\mu}^{2}\right) .
\end{gathered}
$$

Similarly,

$$
\begin{aligned}
& \sum_{\mu, \nu, \lambda \in I_{q}} E\left(\left|Z_{\mu}-p_{\mu}\right| \cdot\left|Z_{\nu}-p_{\nu}\right| \cdot\left|Z_{\lambda}-p_{\lambda}\right|\right) \\
& \leqq\left.\leqq \sum_{I_{q}} E\left(\left|Z_{\mu}-p_{\mu}\right|^{3}\right) E\left(\left|Z_{\nu}-p_{\nu}\right|^{3}\right) E\left(\left|Z_{\lambda}-p_{\lambda}\right|^{3}\right)\right\}^{1 / 3} \\
& \leqq \sum_{I_{q}} E\left(\left|Z_{\mu}-p_{\mu}\right|^{3}\right) \quad=e_{q}+O\left(\sum_{I_{q}} p_{\mu}^{2}\right) .
\end{aligned}
$$

Thus (49) reduces to the triviality

$$
\sum_{q=2}^{\kappa} e_{q}+O(1)=o\left\{\left(\sum_{q=2}^{\kappa} e_{q}\right)^{3 / 2}\right\} .
$$


To complete the proof of Theorem 3, we must show that the hypotheses of Theorem 4 are satisfied when $Z_{n}=Y_{n}, p_{n}=\operatorname{Pr}\left\{\varepsilon_{n}\right\}$. We know that

$$
2 f(n) \leqq p_{n} \leqq 8 f(n) \text {, }
$$

and hence, from the hypotheses of Theorem 3, we obtain (38) and (39). Since the $Y_{n}$ are $2 m$-dependent, we can rewrite (40) in the form

$$
\sum_{i=1}^{\infty} \sum_{j=1}^{2 m}\left|\operatorname{Cov}\left(Y_{i}, Y_{i+j}\right)\right|<\infty
$$

Now if $j>k_{n}$, then $Y_{i}$ and $Y_{i+j}$ are independent, and their covariance is 0 . If $i \leqq j \leqq k_{n}$, then

$$
\begin{aligned}
\left|\operatorname{Cov}\left(Y_{i}, Y_{i+j}\right)\right| & =\left|E\left(Y_{i} Y_{i+j}\right)-E\left(Y_{i}\right) E\left(Y_{i+j}\right)\right| \\
& =\left|\operatorname{Pr}\left\{Y_{i}=Y_{i+j}=1\right\}-\operatorname{Pr}\left\{Y_{i}=1\right\} \cdot \operatorname{Pr}\left\{Y_{i+j}=1\right\}\right| \\
& \leqq\left(r_{n+1} \cdots r_{n+k_{n}}\right)^{-1}+8 f(i) f(i+j),
\end{aligned}
$$

and the convergence of (50) follows from (32).

\section{A strong theorem.}

Theorem 5. Let $\left\{R_{n}\right\}$ and $f(n)$ satisfy the hypotheses of Theorem 3. Then for almost all $x$, the number of integers $m \leqq n$, for which $\left\langle R_{m} x\right\rangle\langle f(m)$, is asymptotic to

$$
2 \sum_{k=1}^{n} f(k)
$$

As in the proof of Theorem 3 , it suffices to prove the theorem with $S_{n}$ replaced by $T_{n}=\sum_{1}^{n} Y_{k}$, and to suppose that $f(n)>n^{-2}$, so that the $Y_{k}$ are $2 m$ dependent. We write

$$
\begin{aligned}
T_{n} & =\sum^{*} Y_{2 m v+1}+\sum^{*} Y_{2 m v+2}+\cdots+\sum^{*} Y_{2 m v+2 m} \\
& =T_{n}^{(1)}+T_{n}^{(2)}+\cdots+T_{n}^{(2 m)}
\end{aligned}
$$

where each summation extends over those $\nu$ for which the subscripts are not larger than $n$. The terms in $T_{n}^{(j)}$ are independent and uniformly bounded, and

$$
E\left(T_{n}^{(j)}\right)=2 \sum^{*} f(2 m \nu+j), \quad \operatorname{Var}\left(T^{(j)}\right)=2 \sum^{*} f(2 m \nu+j)+O(1) .
$$

Hence Kolmogorov's version of the law of the iterated logarithm [8] implies that for $1 \leqq j \leqq 2 m$,

$$
\operatorname{Pr}\left\{\limsup _{n \rightarrow \infty} \frac{\left|T_{n}^{(j)}-2 \sum^{*} f(2 m \nu+j)\right|}{2\left(\sum^{*} f(2 m \nu+j) \cdot \log \log \sum^{*} f(2 m \nu+j)\right)^{1 / 2}}=1\right\}=1 ;
$$

and it follows from these equations that 


$$
\operatorname{Pr}\left\{\left|T_{n}-2 \sum_{k=1}^{n} f(k)\right|=O\left(\sum_{j=1}^{2 m}\left(\sum^{*} f(2 m \nu+j) \cdot \log _{2} \sum^{*} f(2 m \nu+j)\right)^{1 / 2}\right)\right\}=1,
$$

and the theorem is a weak consequence of this result.

Note added in proof.

I. There is a strong version of Theorem 1:

Under the hypotheses of Theorem 1 , the number of solutions $m \leqq n$ of the inequality $\langle m x\rangle\langle g(m)$ is asymptotic to

for almost all $x$.

$$
\frac{12}{\pi^{2}} \sum_{k=1}^{n} g(k)
$$

The proof depends on a strong law of large numbers for dependent variables, due to Lévy [10, p. 253]: Under the hypotheses of Lemma 3,

$$
\operatorname{Pr}\left\{\lim _{t \rightarrow \infty} \frac{S(t)}{t^{1 / 2+\epsilon}}=0\right\}=1
$$

for every positive constant $\epsilon$. Using this in place of Lemma 3, we obtain a strong analogue of Lemma 2, to the effect that for $\epsilon>0$,

$$
\operatorname{Pr}\left\{W_{n}-(\log 2)^{-1} \sum_{1}^{n} f(k)=o\left(\left(\sum_{1}^{n} f(k)\right)^{1 / 2+\epsilon}\right)\right\}=1,
$$

and thereafter the proof parallels that of Theorem 1 .

II. It has been pointed out to me that Lemma 3 is not immediately applicable in the proof of Lemma 2, since $E_{k}\left(V_{k}\right)$, in the equation preceding (8), means $E\left(V_{k}\right.$, given $\left.a_{0}, \cdots, a_{k}\right)$ and not $E\left(V_{k}\right.$, given $\left.V_{0}, \cdots, V_{k-1}\right)$, and it is possible that $V_{k-1}$, for example, is not uniquely determined by $a_{0}, \cdots, a_{k}$. But in order for this to be the case it is necessary, since $\left|q_{k-1} x-p_{k-1}\right|$ $=\left(q_{k-1} x+q_{k-2}\right)^{-1}$ and $a_{k}=\left[x_{k}\right]$, that

$$
\frac{1}{q_{k-1}\left(a_{k}+1\right)+q_{k-2}}<\frac{f(k-1)}{q_{k-1}}<\frac{1}{q_{k-1} a_{k}+q_{k-2}} .
$$

This happens only if

$$
a_{k}=\left[\frac{1}{f(k-1)}-\frac{q_{k-2}}{q_{k-1}}\right] .
$$

The difficulty vanishes, therefore, if we prove the following theorem, and exclude from the beginning the exceptional set mentioned in it (taking $b=1$ and $h(k)=1 / f(k-1))$ :

Let $h$ be a real-valued function on the positive integers, with $h(k)>c k$ for some positive constant $c$. Then for every positive constant $b$, the set of $x$, for which the inequality $\left|a_{k}-h(k)\right|<b$ has infinitely many solutions, has measure zero. 
Put $F_{k}(t)=\operatorname{Pr}\left\{x_{k}<t\right\}$; then Lévy's form of the Gauss-Kuzmin theorem [10, pp. 298-306] asserts that for some $g$ with $0<g<1$,

$$
\left|F_{k}(t)-\frac{1}{\log 2} \log \frac{2 t}{t+1}\right|<g^{k-1}
$$

for all $t>1$ and all positive integers $k$. Now the inequality $\left|a_{k}-h(k)\right|<b$ is equivalent to

$$
h(k)-b<x_{k}<h(k)+b+1,
$$

and we have

$$
\begin{aligned}
\lim _{k \rightarrow \infty} \operatorname{Pr}\left\{h(k)-b<x_{k}\right. & <h(k)+b+1\} \\
& <\frac{1}{\log 2} \log \left(\frac{2(h(k)+b+1)}{h(k)+b+2} \cdot \frac{h(k)-b+1}{2(h(k)-b)}\right)+2 g^{k-1} \\
& =\frac{1}{\log 2} \log \frac{2 h^{2}(k)+4 h(k)-2\left(b^{2}-1\right)}{2 h^{2}(k)+4 h(k)-2\left(b^{2}+b\right)}+2 g^{k-1} \\
& =\frac{1}{\log 2} \log \left(1+O\left(h^{-2}(k)\right)\right)+2 g^{k-1}=O\left(h^{-2}(k)\right)+2 g^{k-1} .
\end{aligned}
$$

Hence the probabilities of the inequalities in question form the terms of a convergent series, and the required result follows from the Borel-Cantelli lemma.

\section{REFERENCES}

1. H. Cramér, Methods of mathematical statistics, Princeton University Press, 1946.

2. J. L. Doob, Stochastic processes, New York, J. Wiley and Sons, 1953.

3. W. Feller, Probability theory and its applications, New York, J. Wiley and Sons, 1950.

4. G. H. Hardy, J. E. Littlewood and G. Polya, Inequalities, Cambridge University Press, 1934.

5. W. Hoeffding and H. Robbins, The central limit theorem for dependent random variables, Trans. Amer. Math. Soc. vol. 15 (1948) pp. 773-780.

6. A. Ya. Khinchin, Einige Sätze über Kettenbrüche, mit Anwendungen auf die Theorie der diophantischen Approximationen, Math. Ann. vol. 92 (1924) pp. 115-125.

7. — Zur metrischen Kettenbruchtheore, Comp. Math. vol. 3 (1936) pp. 276-285.

8. A. Kolmogorov, Über das Gesetz des iterierten Logarithmus, Math. Ann. vol. 101 (1929) pp. 126-135.

9. P. Lévy, Sur le développement en fraction continue d'un nombre choisie au hasard, Comp. Math. vol. 3 (1936) pp. 286-303.

10. — Théorie de l'addition des variables aléatoires, Paris, Gauthier-Villars, 1937.

UNIVERSITY OF Michigan,

ANN ARbor, Mich. 